Research Article

\title{
Risk Factors Associated with Late Failure of Noninvasive Ventilation in Patients with Chronic Obstructive Pulmonary Disease
}

\author{
Tao Chen, Linfu Bai, Wenhui Hu, Xiaoli Han, and Jun Duan \\ The Department of Respiratory and Critical Care Medicine, The First Affiliated Hospital of Chongqing Medical University, \\ Chongqing, China \\ Correspondence should be addressed to Jun Duan; duanjun412589@163.com
}

Received 19 August 2020; Revised 25 September 2020; Accepted 29 September 2020; Published 14 October 2020

Academic Editor: Jack Kastelik

Copyright (c) 2020 Tao Chen et al. This is an open access article distributed under the Creative Commons Attribution License, which permits unrestricted use, distribution, and reproduction in any medium, provided the original work is properly cited.

Background. Risk factors for noninvasive ventilation (NIV) failure after initial success are not fully clear in patients with acute exacerbation of chronic obstructive pulmonary disease (COPD). Methods. Patients who received NIV beyond $48 \mathrm{~h}$ due to acute exacerbation of COPD were enrolled. However, we excluded those whose $\mathrm{pH}$ was higher than 7.35 or $\mathrm{PaCO}_{2}$ was less than $45 \mathrm{mmHg}$ which was measured before NIV. Late failure of NIV was defined as patients required intubation or died during NIV after initial success. Results. We enrolled 291 patients in this study. Of them, 48 (16\%) patients experienced late NIV failure (45 received intubation and 3 died during NIV). The median time from initiation of NIV to intubation was 4.8 days (IQR: 3.4-8.1). Compared with the data collected at initiation of NIV, the heart rate, respiratory rate, $\mathrm{pH}$, and $\mathrm{PaCO}_{2}$ significantly improved after $1-2 \mathrm{~h}$ of NIV both in the NIV success and late failure of NIV groups. Nosocomial pneumonia (odds ratio $(\mathrm{OR})=75,95 \%$ confidence interval (CI): 11-537), heart rate at initiation of NIV $(1.04,1.01-1.06$ beat per min), and $\mathrm{pH}$ at $1-2 \mathrm{~h}$ of NIV (2.06, 1.41-3.00 per decrease of 0.05 from 7.35) were independent risk factors for late failure of NIV. In addition, the Glasgow coma scale $\left(\mathrm{OR}=0.50,95 \% \mathrm{CI}: 0.34-0.73\right.$ per one unit increase) and $\mathrm{PaO}_{2} / \mathrm{FiO}_{2}(0.992,0.986-0.998$ per one unit increase) were independent protective factors for late failure of NIV. In addition, patients with late failure of NIV had longer ICU stay (median 9.5 vs. 6.6 days) and higher hospital mortality (92\% vs. 3\%) compared with those with NIV success. Conclusions. Nosocomial pneumonia; heart rate at initiation of NIV; and consciousness, acidosis, and oxygenation at 1-2 h of NIV were associated with late failure of NIV in patients with COPD exacerbation. And, late failure of NIV was associated with increased hospital mortality.

\section{Introduction}

Chronic obstructive pulmonary disease (COPD) is the fourth leading cause of death [1]. Acute exacerbations of COPD are responsible for more than 600,000 hospitalizations annually and result in direct costs of more than $\$ 20$ billion in the United States [2]. Noninvasive ventilation (NIV) as an effective intervention has been used to manage patients with acute exacerbation of COPD for decades. It improves $\mathrm{pH}$, reduces respiratory rate, reduces $\mathrm{PaCO}_{2}$, and subsequently reduces intubation rate and mortality $[3,4]$. Because of these advantages, use of NIV in patients with acute exacerbation of COPD has continuously increased in recent years $[5,6]$. Moreover, current guidelines strongly recommend NIV to be used in patients with acute exacerbation of COPD $[7,8]$.

In spite of benefits from NIV in patients with acute exacerbation of COPD, late failure of NIV after initial improvement is not rare. It ranges from $8 \%$ to $23 \%$ [9-12]. The reasons for early failure of NIV (failure occurred at initial $48 \mathrm{~h}$ of NIV) have been widely discussed in patients with acute exacerbation of COPD [13-18]. However, only few studies have reported the reasons for late failure of NIV $[9,12,19]$. Because of small sample sizes, these studies only identified 
poor sleep, delirium, metabolic complications, and functional limitation were associated with late failure of NIV. Thus, we aimed to find other potential risk factors for late failure of NIV in patients with acute exacerbation of COPD.

\section{Methods}

We performed an observational study in a respiratory ICU of a teaching hospital from January 2012 to December 2015. The study protocol was approved by our ethics committee and the institutional review board (the First Affiliated Hospital of Chongqing Medical University). Because of the observational nature, the informed consents were waived.

Patients who were admitted to our ICU for NIV as a firstline intervention because of acute exacerbation of COPD were screened for eligibility. COPD was diagnosed based on the guideline developed by our Respiratory Disease Committee, Chinese Medical Association in 2002 [20]. We enrolled the patients whose $\mathrm{pH}$ was less than 7.35 and $\mathrm{PaCO}_{2}$ was more than $45 \mathrm{mmHg}$ which were measured before NIV. However, we excluded those whose NIV was terminated because of clinical improvement, requirement of intubation, or death within $48 \mathrm{~h}$ of NIV. Late failure of NIV was defined as intubation or death during NIV after initial success [9].

In our department, NIV was managed by attending physicians, respiratory therapists, and nurses as the protocol reported previously [21]. The face mask (ZS-MZA Face Mask; Shanghai Zhongshan Medical Technology Co., Shanghai, China) was the first choice for NIV (BiPAP Vision or Respironics V60). Patients were positioned at $30^{\circ}$ to $45^{\circ}$ to avoid aspiration, if there were no contraindications to this positioning. Bi-level positive airway pressure (S/T mode) was used for all patients. Expiratory positive airway pressure was initially set at $4 \mathrm{cmH}_{2} \mathrm{O}$ and titrated according to the flow curve to ensure that expiratory flow reached zero prior to inspiration or diminished ineffective efforts. However, it was limited to less than $12 \mathrm{cmH}_{2} \mathrm{O}$. Inspiratory positive airway pressure was set at $8 \mathrm{cmH}_{2} \mathrm{O}$ and increased by increments of $2 \mathrm{cmH}_{2} \mathrm{O}$ to obtain a tidal volume of more than $6 \mathrm{~mL} / \mathrm{kg}$ or to the maximum tolerated level for each patient. The inspiratory positive airway pressure was limited to less than $25 \mathrm{cmH}_{2} \mathrm{O}$. The fraction of inspired oxygen was set to maintain $\mathrm{SpO}_{2}$ at around 95\%. Humidification was provided by a heated humidifier. If humidification was inadequate, intermittent drinking was allowed. If respiratory failure was reversed, disconnection of NIV equipment was performed per hospital protocol [22].

Intubation was performed referencing the criteria as follows (one major criterion or at least two minor criteria), but it was determined at the discretion of the attending physicians [21]. Major criteria were (1) respiratory arrest, (2) loss of consciousness, (3) hemodynamic instability without response to fluids and vasoactive agents, (4) inability to correct dyspnea, (5) development of conditions necessitating intubation to protect the airway or to manage copious tracheal secretions, and (6) $\mathrm{PaO}_{2} / \mathrm{FiO}_{2}$ below $100 \mathrm{mmHg}$. Minor criteria were (1) respiratory rate more than 35 breaths/min, (2) blood $\mathrm{pH}$ less than 7.30, (3) persistent tachycardia, (4) persistent activation of accessory respiratory muscles, and (5) $\mathrm{PaO}_{2} / \mathrm{FiO}_{2}$ below $150 \mathrm{mmHg}$.

Nosocomial pneumonia was diagnosed by the methods we reported previously [23]. It was suspected if a patient had a radiographic infiltrate that was new or progressive, along with clinical findings suggesting infection, including new onset of fever, purulent sputum, leukocytosis, and decline in oxygenation. In patients with suspected pneumonia, respiratory tract culture was performed. Samples were obtained by coughing, nasotracheal suction, a protected specimen brush, or bronchoalveolar lavage. Nosocomial pneumonia was confirmed by positive culture and clinical presentations.

Data were analyzed by statistical software (SPSS 17.0; SPSS, Chicago, IL, USA) and reported as mean and standard deviation or median and interquartile range when appropriate. Normally distributed continuous variables were analyzed with the independent-sample $t$-test. Abnormally distributed continuous variables were analyzed with the Mann-Whitney $U$ test. Categorical variables were analyzed by the chi-square or Fisher's exact test when appropriate. Within groups, a paired-sample $t$-test was used to analyze the data collected at NIV initiation and 1-2 h of NIV. Kaplan-Meier curves were used to analyze the proportions of intubation in patients with late failure of NIV. Independent risk factors for late failure of NIV were identified by multivariate logistic regression analysis. $p<0.05$ was considered significant.

\section{Results}

We enrolled 291 patients in this study. After 48 h of NIV, 45 patients experienced intubation. The median time from initiation of NIV to intubation was 4.8 days (interquartile range (IQR): 3.4-8.1) (Figure 1). In addition, 3 patients reached the criteria of intubation. In spite of attending physicians, they did not benefit from intubation and continuous use of NIV. Finally the 3 patients died during NIV. Thus, a total of 48 patients (16\%) experienced late NIV failure after initial success.

Patients with NIV success were younger than those with late failure of NIV (71 \pm 10 vs. $76 \pm 9$ years, $p=0.01)(\mathrm{Ta}-$ ble 1). They also had lower APACHE II score (17 \pm 6 vs. $21 \pm 5, \quad p<0.01)$ and lower proportion of nosocomial pneumonia ( $0.8 \%$ vs. $14.6 \%, p<0.01)$. At initiation of NIV, there were no differences in respiratory rate, $\mathrm{pH}$, and $\mathrm{PaO}_{2} /$ $\mathrm{FiO}_{2}$ between patients with NIV success and late failure of NIV. However, the patients with NIV success had lower respiratory rate $(23 \pm 4$ vs. $25 \pm 7$ breaths $/ \mathrm{min}, p=0.02)$, higher $\mathrm{pH}(7.34 \pm 0.06$ vs. $7.31 \pm 0.08, p=0.02)$, and higher $\mathrm{PaO}_{2} / \mathrm{FiO}_{2} \quad(221 \pm 81$ vs. $183 \pm 76 \mathrm{mmHg}, \quad p<0.01)$ after 1-2 h of NIV compared with those who experienced late failure of NIV. Compared with the variables collected at NIV initiation, respiratory rate, heart rate, $\mathrm{pH}$, and $\mathrm{PaCO}_{2}$ collected at $1-2 \mathrm{~h}$ of NIV significantly improved both in NIV success and late failure of NIV groups (Figure 2). However, the respiratory rate, $\mathrm{pH}, \mathrm{PaCO}_{2}$, and $\mathrm{PaO}_{2} / \mathrm{FiO}_{2}$ improved 


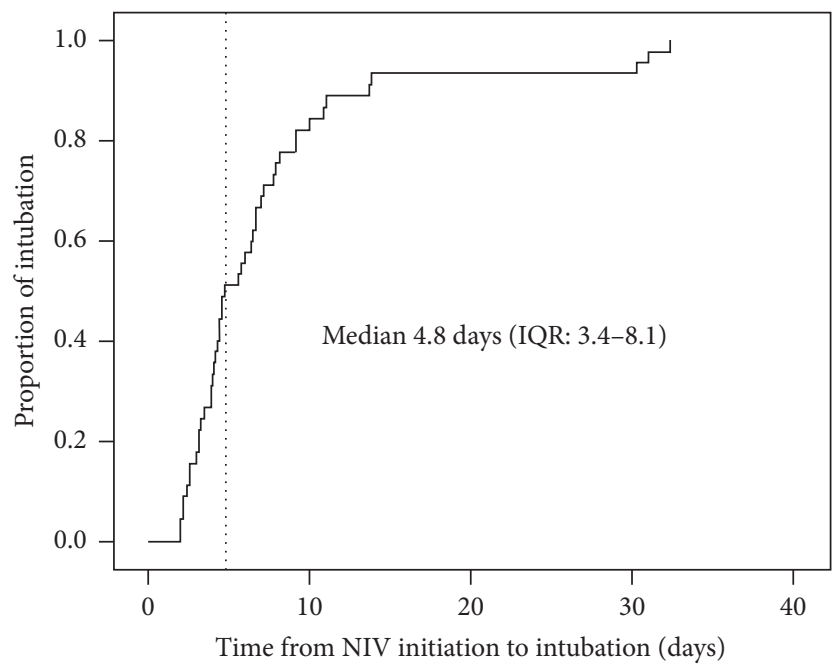

FIGURE 1: Proportion of intubation in patients with late failure of NIV.

TABLE 1: Baseline characteristics of patients who had NIV success or late failure of NIV.

\begin{tabular}{|c|c|c|c|}
\hline & $\begin{array}{c}\text { NIV success } \\
N=243(84 \%)\end{array}$ & $\begin{array}{l}\text { Late failure of NIV } \\
\qquad N=48(16 \%)\end{array}$ & $p$ \\
\hline Age, years & $71 \pm 10$ & $76 \pm 9$ & $0.01^{*}$ \\
\hline Male/female & $186 / 57$ & $33 / 15$ & 0.27 \\
\hline APACHE II score & $17 \pm 6$ & $21 \pm 5$ & $<0.01^{*}$ \\
\hline Nosocomial pneumonia during NIV & $2(0.8 \%)$ & $7(14.6 \%)$ & $<0.01^{*}$ \\
\hline \multicolumn{4}{|l|}{ Data collected at NIV initiation } \\
\hline GCS & $14.6 \pm 1.1$ & $13.8 \pm 1.7$ & $<0.01^{*}$ \\
\hline Respiratory rate, breaths/min & $29 \pm 5$ & $29 \pm 6$ & 0.85 \\
\hline Heart rate, beats/min & $110 \pm 19$ & $120 \pm 26$ & $<0.01^{*}$ \\
\hline MAP, $\mathrm{mmHg}$ & $102 \pm 17$ & $94 \pm 18$ & $0.01^{*}$ \\
\hline $\mathrm{pH}$ & $7.26 \pm 0.06$ & $7.27 \pm 0.06$ & 0.80 \\
\hline $\mathrm{PaCO}_{2}, \mathrm{mmHg}$ & $81 \pm 19$ & $73 \pm 23$ & $0.01^{*}$ \\
\hline $\mathrm{PaO}_{2} / \mathrm{FiO}_{2}, \mathrm{mmHg}$ & $192 \pm 103$ & $188 \pm 99$ & 0.79 \\
\hline \multicolumn{4}{|l|}{ Data collected at $1-2 \mathrm{~h}$ of NIV } \\
\hline GCS & $14.8 \pm 0.7$ & $13.9 \pm 1.9$ & $<0.01^{*}$ \\
\hline Respiratory rate, breaths/min & $23 \pm 4$ & $25 \pm 7$ & $0.02^{*}$ \\
\hline Heart rate, beats/min & $100 \pm 18$ & $109 \pm 22$ & $<0.01^{*}$ \\
\hline $\mathrm{MAP}, \mathrm{mmHg}$ & $91 \pm 14$ & $90 \pm 16$ & 0.50 \\
\hline $\mathrm{pH}$ & $7.34 \pm 0.06$ & $7.31 \pm 0.08$ & $0.02^{*}$ \\
\hline $\mathrm{PaCO}_{2}, \mathrm{mmHg}$ & $70 \pm 18$ & $68 \pm 21$ & 0.33 \\
\hline $\mathrm{PaO}_{2} / \mathrm{FiO}_{2}, \mathrm{mmHg}$ & $221 \pm 81$ & $183 \pm 76$ & $<0.01^{*}$ \\
\hline
\end{tabular}

NIV $=$ noninvasive ventilation; GCS $=$ Glasgow coma scale; MAP $=$ mean arterial pressure ${ }^{*} p<0.05$ for NIV success vs. late failure of NIV.

faster in the NIV success group than those in the late failure of NIV group (Figure 3).

In the multivariate logistic regression analysis, we identified that nosocomial pneumonia (odds ratio $(\mathrm{OR})=$ $75,95 \%$ confidence interval (CI): 11-537), heart rate at initiation of NIV $(1.04,1.01-1.06$ beat per min), and $\mathrm{pH}$ at $1-2 \mathrm{~h}$ of NIV $(2.06,1.41-3.00$ per decrease of 0.05 from 7.35$)$ were independent risk factors for late failure of NIV (Table 2). We also found that the Glasgow coma scale $(\mathrm{OR}=0.50,95 \% \mathrm{CI}: 0.34-0.73$ per one unit increase $)$ and $\mathrm{PaO}_{2} / \mathrm{FiO}_{2}$ (0.992, 0.986-0.998 per one unit increase) were independent protective factors for late failure of NIV.

Outcomes between patients with NIV success and late failure are summarized in Table 3. There were no differences in duration of NIV and the length of stay in the hospital between the two groups. However, the patients with NIV success had shorter length of stay in the ICU (median 6.6, IQR: $4.9-9.8$ vs. 9.5, 5.7-13.8, $p=0.02$ ) and lower hospital mortality ( $3 \%$ vs. $92 \%, p<0.01)$ than those with late failure of NIV.

\section{Discussion}

The current study found the incidence of late failure of NIV was $16 \%$ in patients with acute exacerbation of COPD with a relatively large sample size. Although some clinical variables improved both in the NIV success and late failure of NIV groups, the variables in the NIV success group improved faster than those in the late failure of NIV group. Nosocomial pneumonia; heart rate at initiation of NIV; and 


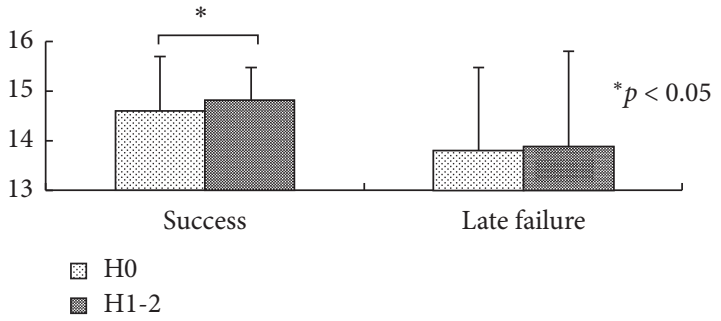

(a)

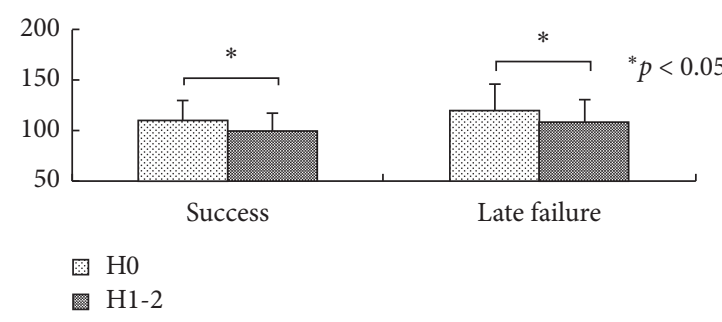

(c)

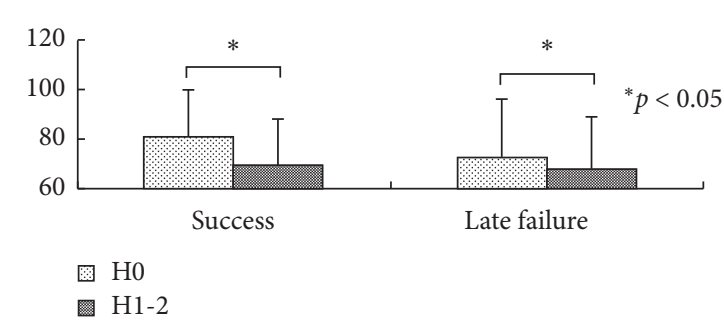

(e)

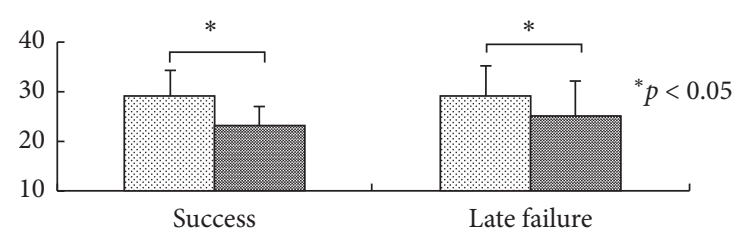

图 $\mathrm{H} 0$

目 $\mathrm{H} 1-2$

(b)

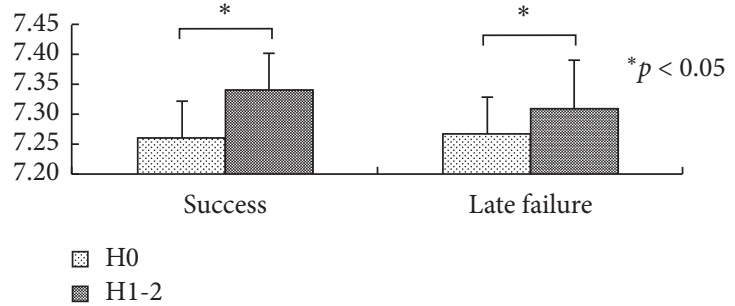

(d)

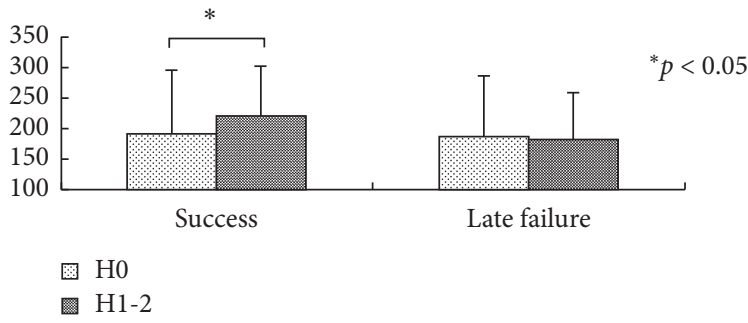

(f)

FIGURE 2: Comparisons between initiation and 1-2 h of NIV: (a) GCS, (b) respiratory rate (breaths/min), (c) heart rate (beats/min), (d) pH, (e) $\mathrm{PaCO}_{2}$ (mmHg), and (f) $\mathrm{PaO}_{2} / \mathrm{FiO}_{2}(\mathrm{mmHg})$.

consciousness, acidosis, and oxygenation at 1-2 h of NIV were associated with late failure of NIV. In addition, late failure of NIV was associated with increased hospital mortality.

The mortality in patients with late failure of NIV was $68 \%$ in Moretti's study and $80 \%$ in Carratu's study $[9,12]$. In our study, the mortality was $92 \%$, which was higher than the value reported by previous studies. We noted that most of the patients experienced NIV failure within 15 days of NIV in our study. However, some cases experienced NIV failure beyond 30 days. From 15 to 30 days of NIV, there was no NIV failure. It indicates that some patients had significantly impaired respiratory function and required prolonged noninvasive ventilation. In addition, longer exposure in the ICU is associated with a higher incidence of nosocomial pneumonia. These reasons contribute much to hospital mortality.

Previous studies reported that patients with late failure of NIV had higher APACHE II score, higher heart rate, lower GCS, and lower blood pressure compared with successful ones [12, 19, 24]. Our study also found similar results. Different from previous studies, we found nosocomial pneumonia was an independent risk factor for late failure of NIV. It reminds us that nosocomial pneumonia played an important role in late failure of NIV. Among the NIV patients who experienced nosocomial pneumonia in our study, NIV failure occurred in $78 \%$ of cases. Thus, prevention of nosocomial pneumonia in NIV patients was as important as in those who received invasive mechanical ventilation.

Both in the NIV success and late failure of NIV groups, most of the clinical variables significantly improved after 1-2 h of NIV. However, the respiratory rate, $\mathrm{pH}, \mathrm{PaCO}_{2}$, and $\mathrm{PaO}_{2} / \mathrm{FiO}_{2}$ improved faster in the NIV success group than those in the late failure of NIV group. These results are new findings compared with previous studies $[9,12,19,24]$. These data indicate that the patients in the late failure of NIV group responded not so well than those who experienced NIV success. That may be the reason for initial improvement but later failure in the late failure of NIV group.

Our study has several limitations. We found nosocomial pneumonia was associated with late failure of NIV in a patient with acute exacerbation of COPD. However, we only enrolled 9 patients with nosocomial pneumonia. The small sample size may skew this result. Thus, the result is required to be validated with a larger sample size. Secondly, this study was only performed in a respiratory ICU. The single-center study also limited the results to extrapolate to other centers. Thirdly, patients who received intubation later were 


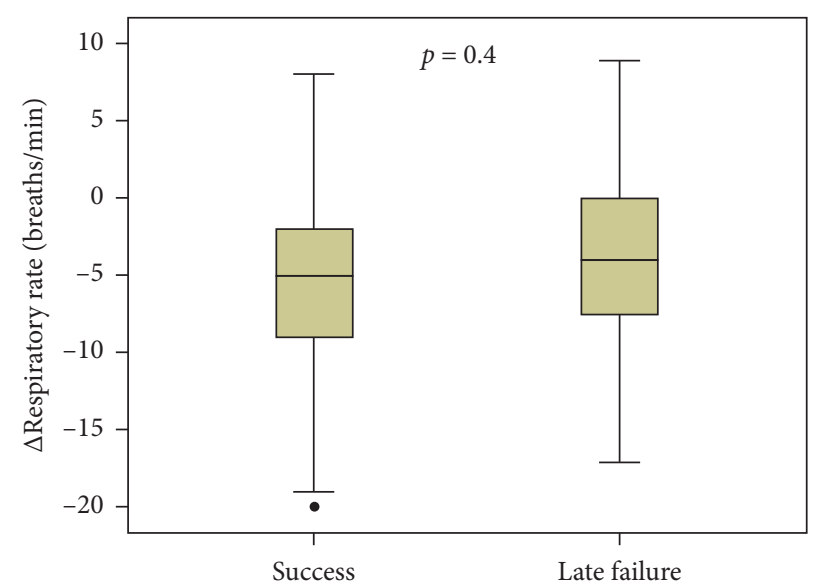

(a)

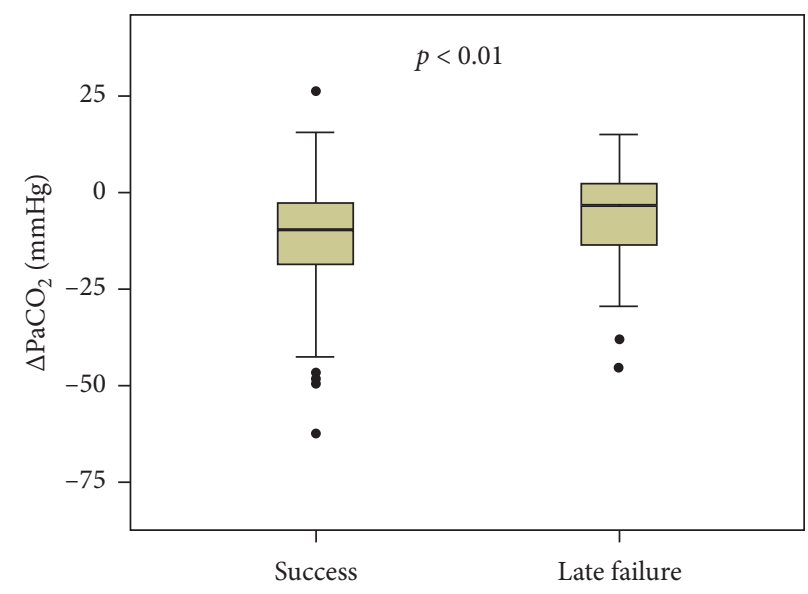

(c)

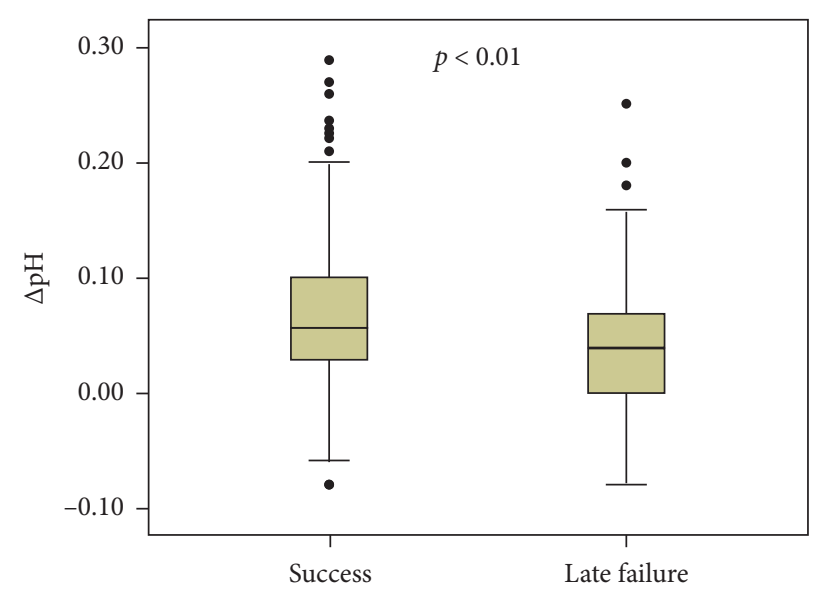

(b)

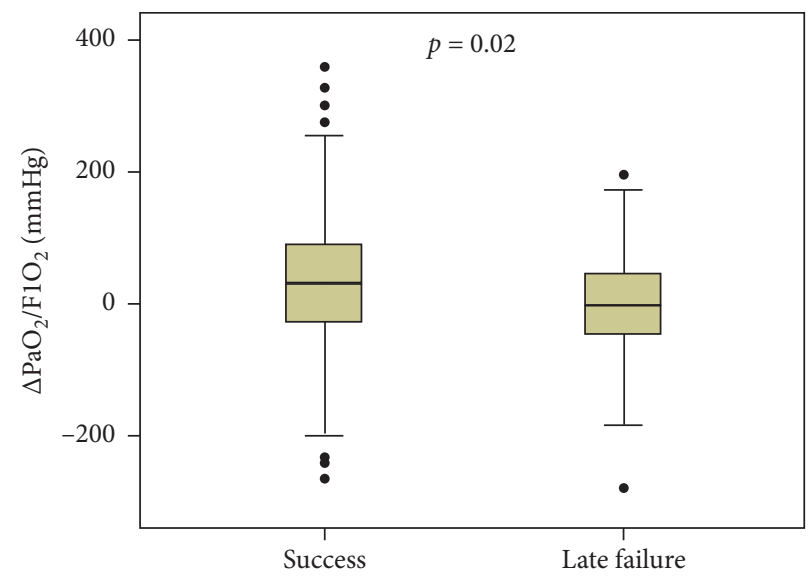

(d)

FIgURE 3: Changes of vital signs from initiation to $1-2 \mathrm{~h}$ of NIV.

TABLE 2: Univariate and multivariate analysis of risk factors associated with late failure of NIV.

\begin{tabular}{|c|c|c|c|c|}
\hline & \multicolumn{2}{|c|}{ Univariate analysis } & \multicolumn{2}{|c|}{ Multivariate analysis } \\
\hline & OR $(95 \% \mathrm{CI})$ & $p$ & OR $(95 \% \mathrm{CI})$ & $p$ \\
\hline Age, years & $1.05(1.01-1.08)$ & $<0.01$ & - & - \\
\hline APACHE II score & $1.11(1.05-1.18)$ & $<0.01$ & - & - \\
\hline Nosocomial pneumonia during NIV & $21(4-103)$ & $<0.01$ & $75(11-537)$ & $<0.01$ \\
\hline \multicolumn{5}{|l|}{ Data collected at NIV initiation } \\
\hline GCS & $0.70(0.57-0.86)$ & $<0.01$ & - & - \\
\hline Heart rate, beats/min & $1.02(1.01-1.04)$ & $<0.01$ & $1.04(1.01-1.06)$ & $<0.01$ \\
\hline $\mathrm{MAP}, \mathrm{mmHg}$ & $0.98(0.96-0.99)$ & $<0.01$ & - & - \\
\hline $\mathrm{PaCO}_{2}, \mathrm{mmHg}$ & $0.98(0.97-1.00)$ & 0.01 & - & - \\
\hline \multicolumn{5}{|l|}{ Data collected at $1-2 \mathrm{~h}$ of NIV } \\
\hline GCS & $0.50(0.36-0.70)$ & $<0.01$ & $0.50(0.34-0.73)$ & $<0.01$ \\
\hline Respiratory rate, breaths/min & $1.08(1.01-1.14)$ & 0.02 & - & - \\
\hline Heart rate, beats/min & $1.03(1.01-1.04)$ & 0.01 & - & - \\
\hline $\mathrm{pH}$ at $1-2 \mathrm{~h}$ of NIV, per decrease of 0.05 from 7.35 & $1.67(1.26-2.25)$ & $<0.01$ & $2.06(1.41-3.00)$ & $<0.01$ \\
\hline $\mathrm{PaO}_{2} / \mathrm{FiO}_{2}, \mathrm{mmHg}$ & $0.993(0.988-0.998)$ & $<0.01$ & $0.992(0.986-0.998)$ & 0.01 \\
\hline
\end{tabular}

$\mathrm{OR}=$ odds ratio; $\mathrm{CI}=$ confidence interval; $\mathrm{NIV}=$ noninvasive ventilation; $\mathrm{GCS}=$ Glasgow coma scale; $\mathrm{MAP}=$ mean arterial pressure . 
TABLE 3: Outcomes between patients with NIV success and late failure.

\begin{tabular}{|c|c|c|c|}
\hline & $\begin{array}{l}\text { NIV success, } \\
N=243(84 \%)\end{array}$ & $\begin{array}{l}\text { Late failure of NIV, } \\
\qquad N=48(16 \%)\end{array}$ & $p$ \\
\hline Duration of NIV (median (IQR)), days & $5.0(3.5-7.7)$ & $5.2(3.4-9.0)$ & 0.52 \\
\hline Duration of ICU stay (median (IQR)), days & $6.6(4.9-9.8)$ & $9.5(5.7-13.8)$ & $0.02^{*}$ \\
\hline Duration of hospital stay (median (IQR)), days & $13.0(8.3-19.1)$ & $14.1(9.7-22.7)$ & 0.41 \\
\hline Hospital mortality & $8(3 \%)$ & $44(92 \%)$ & $<0.01^{*}$ \\
\hline
\end{tabular}

$\mathrm{NIV}=$ noninvasive ventilation; $\mathrm{IQR}=$ interquartile range; $\mathrm{ICU}=$ intensive care unit. ${ }^{*} p<0.05$ for NIV success vs. late failure of NIV.

associated with higher mortality $[12,25]$. Therefore, early intubation (e.g., $24 \mathrm{~h}$ of NIV) is an alternative to reduce mortality.

\section{Conclusions}

Nosocomial pneumonia; heart rate at initiation of NIV; and consciousness, acidosis, and oxygenation at 1-2 h of NIV were associated with late failure of NIV in patients with COPD exacerbation. In addition, late failure of NIV was associated with increased hospital mortality.

\section{Abbreviations}

COPD: Chronic obstructive pulmonary disease

NIV: Noninvasive ventilation

GCS: Glasgow coma scale

MAP: Mean arterial pressure

OR: Odds ratio

CI: $\quad$ Confidence interval

IQR: Interquartile range

ICU: Intensive care unit.

\section{Data Availability}

The datasets analyzed during this study are available from the corresponding author upon reasonable request.

\section{Conflicts of Interest}

The authors declare that there are no conflicts of interest in this study.

\section{Authors' Contributions}

Jun Duan conceived the study and joined in study design, study management, data collection, data analysis, and manuscript revision. Tao Chen participated in study design, study management, data collection, data analysis, and manuscript preparation. Linfu Bai, Wenhui $\mathrm{Hu}$, and Xiaoli Han participated in study design, data collection, and manuscript revision. All authors read and approved the final version.

\section{References}

[1] J. Vestbo, S. S. Hurd, A. G. Agustí et al., "Global strategy for the diagnosis, management, and prevention of chronic obstructive pulmonary disease," American Journal of Respiratory and Critical Care Medicine, vol. 187, no. 4, pp. 347-365, 2013.
[2] V. Snow, S. Lascher, C. Mottur-Pilson et al., "Evidence base for management of acute exacerbations of chronic obstructive pulmonary disease," Annals of Internal Medicine, vol. 134, no. 7, pp. 595-599, 2001.

[3] J. V. Lightowler, J. A. Wedzicha, M. W. Elliott et al., "Noninvasive positive pressure ventilation to treat respiratory failure resulting from exacerbations of chronic obstructive pulmonary disease: cochrane systematic review and metaanalysis," British Medical Journal, vol. 326, no. 7382, p. 185, 2003.

[4] F. S. F. Ram, J. Picot, J. Lightowler, and J. A. Wedzicha, "Noninvasive positive pressure ventilation for treatment of respiratory failure due to exacerbations of chronic obstructive pulmonary disease," The Cochrane Database of Systematic Reviews, vol. 1, no. 3, Article ID CD004104, 2004.

[5] D. Chandra, J. A. Stamm, B. Taylor et al., "Outcomes of noninvasive ventilation for acute exacerbations of chronic obstructive pulmonary disease in the United States, 1998-2008," American Journal of Respiratory and Critical Care Medicine, vol. 185, no. 2, pp. 152-159, 2012.

[6] M. S. Mihaela, M.-S. Shieh, P. S. Pekow, N. Hill, M. B. Rothberg, and P. K. Lindenauer, "Trends in mechanical ventilation among patients hospitalized with acute exacerbations of COPD in the United States, 2001 to 2011," Chest, vol. 147, no. 4, pp. 959-968, 2015.

[7] W. Hill and Z. Qing-yuan, "Guideline for mechanical ventilation in patients with acute exacerbation of chronic obstructive pulmonary disease (2007)," Zhongguo Wei Zhong Bing Ji Jiu Yi Xue, vol. 19, pp. 513-518, 2007.

[8] S. P. Keenan, T. Sinuff, K. E. A. Burns et al., "Clinical practice guidelines for the use of noninvasive positive-pressure ventilation and noninvasive continuous positive airway pressure in the acute care setting," Canadian Medical Association Journal, vol. 183, no. 3, pp. E195-E214, 2011.

[9] P. Carratu, P. Bonfitto, S. Dragonieri et al., "Early and late failure of noninvasive ventilation in chronic obstructive pulmonary disease with acute exacerbation," European Journal of Clinical Investigation, vol. 35, no. 6, pp. 404-409, 2005.

[10] A. Carrillo, M. Ferrer, G. Gonzalez-Diaz et al., "Noninvasive ventilation in acute hypercapnic respiratory failure caused by obesity hypoventilation syndrome and chronic obstructive pulmonary disease," American Journal of Respiratory and Critical Care Medicine, vol. 186, no. 12, pp. 1279-1285, 2012.

[11] L. Brochard, J. Mancebo, M. Wysocki et al., "Noninvasive ventilation for acute exacerbations of chronic obstructive pulmonary disease," New England Journal of Medicine, vol. 333, no. 13, pp. 817-822, 1995.

[12] M. Moretti, C. Cilione, A. Tampieri et al., "Incidence and causes of non-invasive mechanical ventilation failure after initial success," Thorax, vol. 55, no. 10, pp. 819-825, 2000.

[13] E. Ozyilmaz, A. O. Ugurlu, and S. Nava, "Timing of noninvasive ventilation failure: causes, risk factors, and potential remedies," BMC Pulmonary Medicine, vol. 14, p. 19, 2014. 
[14] M. Confalonieri, G. Garuti, M. S. Cattaruzza et al., "A chart of failure risk for noninvasive ventilation in patients with COPD exacerbation," European Respiratory Journal, vol. 25, no. 2, pp. 348-355, 2005.

[15] A. Carlucci, J.-C. Richard, M. Wysocki, E. Lepage, and L. Brochard, "Noninvasive versus conventional mechanical ventilation," American Journal of Respiratory and Critical Care Medicine, vol. 163, no. 4, pp. 874-880, 2001.

[16] D. Miller, K. Fraser, I. Murray, G. Thain, and G. P. Currie, "Predicting survival following non-invasive ventilation for hypercapnic exacerbations of chronic obstructive pulmonary disease," International Journal of Clinical Practice, vol. 66, no. 5, pp. 434-437, 2012.

[17] J. Phua, K. Kong, K. H. Lee, L. Shen, and T. K. Lim, "Noninvasive ventilation in hypercapnic acute respiratory failure due to chronic obstructive pulmonary disease vs. other conditions: effectiveness and predictors of failure," Intensive Care Medicine, vol. 31, no. 4, pp. 533-539, 2005.

[18] B. Chakrabarti, R. M. Angus, S. Agarwal, S. Lane, and P. M. A. Calverley, "Hyperglycaemia as a predictor of outcome during non-invasive ventilation in decompensated COPD," Thorax, vol. 64, no. 10, pp. 857-862, 2009.

[19] F. R. Campo, X. Drouot, A. W. Thille et al., "Poor sleep quality is associated with late noninvasive ventilation failure in patients with acute hypercapnic respiratory failure," Critical Care Medicine, vol. 38, no. 2, pp. 477-485, 2010.

[20] Chinese Medical Association Respiratory Disease Committee, "Guideline for the diagnosis and treatment of chronic obstructive pulmonary disease," Chinese Journal of Tuberculosis and Respiratory Disease, vol. 28, pp. 453-460, 2002.

[21] L. Fan, Q. Zhao, Y. Liu, L. Zhou, and J. Duan, "Semiquantitative cough strength score and associated outcomes in noninvasive positive pressure ventilation patients with acute exacerbation of chronic obstructive pulmonary disease," Respiratory Medicine, vol. 108, no. 12, pp. 1801-1807, 2014.

[22] J. Duan, X. Tang, S. Huang, J. Jia, and S. Guo, "Protocoldirected versus physician-directed weaning from noninvasive ventilation," The Journal of Trauma and Acute Care Surgery, vol. 72, no. 5, pp. 1271-1275, 2012.

[23] Z. Zhang and J. Duan, "Nosocomial pneumonia in non-invasive ventilation patients: incidence, characteristics, and outcomes," Journal of Hospital Infection, vol. 91, no. 2, pp. 153-157, 2015.

[24] A. Ciledag, A. Kaya, O. Ercen Diken et al., "The risk factors for late failure of non-invasive mechanical ventilation in acute hypercapnic respiratory failure," Tuberk Toraks, vol. 62, pp. 177-182, 2014.

[25] A. Esteban, F. Frutos-Vivar, N. D. Ferguson et al., "Noninvasive positive-pressure ventilation for respiratory failure after extubation," New England Journal of Medicine, vol. 350, no. 24, pp. 2452-2460, 2004. 